\title{
Catalase and superoxide dismutase activities and the total protein content of protocorm-like bodies of Dendrobium sonia-28 subjected to vitrification
}

\begin{abstract}
Dendrobium sonia-28 is an important ornamental orchid in the Malaysian flower industry. However, the genus faces both low germination rates and the risk of producing heterozygous progenies. Cryopreservation is currently the favoured long-term storage method for orchids with propagation problems. Vitrification, a frequently used cryopreservation technique, involves the application of pretreatments and cryoprotectants to protect and recover explants during and after storage in liquid nitrogen. However, cryopreservation may cause osmotic injuries and toxicity to cryopreserved explants from the use of highly concentrated additives, and cellular injuries from thawing, devitrification and ice formation. Reactive oxygen species (ROS), occurring during dehydration and cryopreservation, may also cause membrane damage. Plants possess efficient antioxidant systems such as the superoxide dismutase (SOD) and catalase (CAT) enzymes to scavenge ROS during low temperature stress. In this study, protocorm-like bodies (PLBs) of Dendrobium sonia-28 were assayed for the total protein content, and both SOD and CAT activities, at each stage of a vitrification exercise to observe for deleterious stages in the protocol. The results indicated that cryopreserved PLBs of Dendrobium sonia-28 underwent excessive post-thawing oxidative stress due to decreased levels of the CAT enzyme at the post-thawing recovery stage, which contributed to the poor survival rates of the cryopreserved PLBs.
\end{abstract}

Keyword: Catalase; Protocorm-like bodies; Superoxide dismutase; Total protein content; Vitrification 